\title{
A POSTERIORI ERROR ESTIMATION IN SENSITIVITY ANALYSIS
}

\author{
Gustavo C. Buscaglia, Claudio Padra \\ Centro Atómico Bariloche, Comisión Nacional de Energía Atómica \\ Centro Atómico Bariloche, 8400, San Carlos de Bariloche, Argentina
}

Raúl A. Feijóo

Laboratório Nacional de Computação Científica

Lauro Müller 455, Rio de Janeiro, 22290.160, Brazil

\begin{abstract}
RESUMEN: Presentamos estimadores a posteriori del error adecuados para refinamiento automático de mallas en la evaluación numérica de la sensibilidad por medio del método de elementos finitos. Se consideran los problemas de difusión (tipo Poisson) y de elasticidad, y se prueba la equivalencia entre el error y el estimador prapuesto. Se expone brevemente la aplicación a la sensibilidad de forma.
\end{abstract}

\begin{abstract}
We present a posteriori error estimators suitable for automatic mesh refinement in the numerical evaluation of sensitivity by means of the finite element method. Both diffusion (Poissontype) and elasticity problems are considered, and the equivalence between the true error and the proposed error estimator is proved. Application to shape sensitivity is briefly addressed.
\end{abstract}

\section{INTRODUCTION}

In the last ten years or so, computational mechanics has evolved, from being essentially an analysis tool, to an integrated part of the design process. Sensitivity analyses, which play a central role in the optimal design of mechanical systems, are becoming standard and can nowadays be found even in some commercial finite element packages (see, e.g., [6]). The recent World Congress STRUCTURAL OPTIMIZATION'93, held in Rio de Janeiro (August $2-6,1993$ ), has been a clear example of the many relevant applications that can be faced with current algorithms and computers.

The purpose of the present paper is to briefly present a posteriori error estimators specifically designed for sensitivity analyses, which can be used to automatically adapt the mesh so as to increase the accuracy of finite element computations. We will concentrate, in this first incursion into the problem, in error estimation for the so-called direct method of evaluating the sensitivity, and the variations in the design will be restricted to the choice of material parameters. Also, the governing equations considered are linear and time-independent. Our scope, however, covers many situations of technological interest, such as steady thermal fields, torsion of cylinders, and linear elastostatics. Error estimation in shape sensitivity analyses is also considered. Further extensions, such as Kirchoff or Reissner-Mindlin plates in bending, are left for future work. Our conclusion from this first work on error estimation in sensitivity analyses is that, if a suitable estimator is available for the error in the solution itself, then it is not difficult to adapt it so as to estimate the error in the derivatives of the solution with respect to the design parameters. 


\section{Preliminaries}

Let us consider first the following model problem

$$
-\nabla \cdot(k(p) \nabla u(p))=f(p)
$$

inside a domain $\Omega$ with (polygonal) boundary $\partial \Omega$. For simplicity, we will assume homogeneous Dirichlet conditions on $\partial \Omega$. The solution $u(p)$, where $p$ is a design real parameter, can be interpreted, e.g., as the temperature. In this case, $k(p)$, with known dependence on $p$, is the thermal conductivity and $f(p)$ a source term. An analogous equation appears in the torsion of a cylinder of arbitrary section and in electrostatics. The variational formulation of $(1)$ is: Find $u(p) \in H_{0}^{1}(\Omega)$ such that

$$
\int_{\Omega} k(p) \nabla u(p) \cdot \nabla v=\int_{\Omega} f(p) v \quad \forall v \in H_{0}^{1}(\Omega)
$$

where $H_{0}^{1}(\Omega)$ is the Hilbert space of square-integrable real functions defined over $\Omega$, with square-integrable derivatives and zero trace on $\partial \Omega$. In this way, we obtain a function $u(p)$ for each $p$ in some real interval. It is known that, if $k$ and $f$ are smooth functions of $p$, so is the solution $u$ itself. We will denote by $\dot{u}(p)$ the derivative of the solution $u$ with respect to $p$, which is again an element of $H_{0}^{1}(\Omega)$ and satisfies the following variational equation

$$
\int_{\Omega} k(p) \nabla \dot{u}(p) \cdot \nabla v=\int_{\Omega} \frac{d f}{d p}(p) v-\int_{\Omega} \frac{d k}{d p}(p) \nabla u(p) \cdot \nabla v \quad \forall v \in H_{0}^{1}(\Omega)
$$

Higher order derivatives of $u$ are obtained in the same way.

We now consider the finite element discretization of (2) and (3). Let $V_{h}$ be a finite-dimensional subspace of $H_{0}^{1}(\Omega)$, and let us define as usual $u_{h}(p)$ as the element of $V_{h}$ which satisfies

$$
\int_{\Omega} k(p) \nabla u_{h}(p) \cdot \nabla v=\int_{\Omega} f(p) v \quad \forall v \in V_{h}
$$

Denoting by $\dot{u}_{h}(p)$ the derivative of $u_{h}$ with respect to $p$, which is also an element of $V_{h}$, we get the following equation for $\dot{u}_{h}(p)$

$$
\int_{\Omega} k(p) \nabla \dot{u}_{h}(p) \cdot \nabla v=\int_{\Omega} \frac{d f}{d p}(p) v-\int_{\Omega} \frac{d k}{d p}(p) \nabla u_{h}(p) \cdot \nabla v \quad \forall v \in V_{h}
$$

where $d k / d p, d f / d p$ stand for the derivatives of $k$ and $f$ (which in general will depend not only on $p$ but also on the space variables) with respect to the parameter $p$.

With the previous definitions, it is well-known [4] that, if $V_{h}$ is a finite-element space and we consider a regular family of triangulations $T_{h}$ parametrized with the diameter $h$ of the largest element, then $\left|u(p)-u_{h}(p)\right|_{1, \Omega}=$ $O\left(h^{k}\right)$, with $k$ the order of the finite element approximation and $|\cdot|_{1, \Omega}$ the seminorm of $H_{0}^{1}(\Omega)$. As shown in [7] under weak hypotheses, a similar estimate holds for all the derivatives with respect to $p$; i.e., for first order sensitivity, $\left|\dot{u}(p)-\dot{u}_{h}(p)\right|_{1, \Omega}=O\left(h^{k}\right)$. It is remarkable that taking derivatives with respect to the parameter implies no loss of accuracy in the spatial approximation.

\section{A posteriori error estimation for $\dot{u}_{h}$}

The a priori error estimates presented above, being of global nature, cannot be used to locally refine the mesh. After a first analysis, with a rough mesh $\mathcal{T}_{h 0}$, a local error indicator $\eta_{K}$ is needed, for each element $K^{*}$ in $T_{h 0}, s 0$ as to decide which elements are too big to accomodate spatial variations in the exact solution and then construct an improved mesh $T_{h 1}$. As the exact solution is obviously not known, $\eta_{K}$ must only depend on the already computed approximate solution, and on the data of the problem. 
For problem (4) several such indicators exist to determine elements where the difference $u-u_{h}$ is largest. We consider here the Babuška-Miller-type [2] error indicator, given by

$$
\eta_{K}^{2}=|K| \int_{K}\left|R\left(u_{h}\right)\right|^{2}+\sum_{i \in \partial K}|l| \int_{i}\left|\left[k^{+} \nabla u_{h}^{+}-k^{-} \nabla u_{h}^{-}\right] \cdot \mathbf{n}\right|^{2}
$$

wherg $|K|$ is the measure of element $K$ and $|l|$ the measure of an edge $l$ belonging to the boundary $\partial K$ of $K$. The supraindices + and - indicate the limits

$$
f^{ \pm}(\mathbf{x})=\lim _{s \rightarrow 0^{+}} f(\mathbf{x} \pm s \mathbf{n}) \quad \mathbf{n}: \text { outward normal }
$$

and $R\left(u_{h}\right)$ is the residual of the differential equation,

$$
R\left(u_{h}\right)=\nabla \cdot\left(k \nabla u_{h}\right)+f
$$

It can be proved that $\sum_{K} \eta_{K}^{2}$ is equivalent to $\left|u-u_{h}\right|_{1, \Omega}^{2}$, and computer experiments have shown that $\eta_{K}$ can be coupled with mesh-refinement or remeshing procedures so as to obtain improved meshes, in which the error is more homogeneously distributed than in the original one.

Remark: It is quite usual to neglect the jump term along element edges in (6), and consider only the residual term. This procedure can only be justified when the exact solution does not contain singularities $[3,8]$.

Let us now turn to the original contents of this section and build an a posteriori error indicator for $\dot{u}-\dot{u}_{h}$. We only sketch the main ideas here, a more rigorous presentation is included in Appendix A.

Consider problems (3) and (5), their left-hand sides are equivalent to those of (2) and (4), and thus one is tempted to use some modification of the error indicator (6). To do this, we must first construct the differential equation for $\dot{u}$, so as to be able to evaluate its residual for (6). Also, the jump term $k^{+} \nabla u_{h}^{+}-k^{-} \nabla u_{h}^{-}$in (6) arises because the differential equation contains the divergence of $k \nabla u$. Integration by parts in (3) immediately yields the desired result

$$
-\nabla \cdot(k(p) \nabla \dot{u}(p))=\nabla \cdot\left(\frac{d k}{d p}(p) \nabla u(p)\right)+\frac{d f}{d p}(p)
$$

At first sight, we are now in position to write down an error indicator $\xi_{K}$ for $\dot{u}-\dot{u}_{h}$, something like

$$
\begin{gathered}
\xi_{K}^{2}=|K| \int_{K}\left|Q\left(\dot{u}_{h}\right)\right|^{2}+\sum_{l \in \partial K}|l| \int_{l}\left|\left[\left(k \nabla \dot{u}_{h}+\frac{d k}{d p} \nabla u_{h}\right)^{+}-\left(k \nabla \dot{u}_{h}+\frac{d k}{d p} \nabla u_{h}\right)^{-}\right] \cdot \mathbf{n}\right|^{2} \\
Q\left(\dot{u}_{h}\right)=\nabla \cdot\left(k \nabla \dot{u}_{h}+\frac{d k}{d p} \nabla u_{h}\right)+\frac{d f}{d p}
\end{gathered}
$$

and we would be almost right, but a closer look at the equation for $\dot{u}_{h}$ (Eq.5) reveals that it is not an approximation to (9) but instead to

$$
-\nabla \cdot\left(k(p) \nabla u^{\prime}(p)\right)=\nabla \cdot\left(\frac{d k}{d p}(p) \nabla u_{h}(p)\right)+\frac{d f}{d p}(p)
$$

where the approximate solution $u_{h}$ appears in the right-hand side. This observation is quite natural: As the exact derivative of the approximate solution $u_{h}(p)\left(u^{\prime}(p)\right.$ in Eq. 11) is not in general an element of $V_{h}$, what we obtain as the solution of (5) is an approrimation to the derivative of the approximate solution. This difficulty can again be easily avoided, just because the difference between the right-hand sides of (9) and (11) can be bounded by some multiple of $\left|u-u_{h}\right|_{1, \Omega}$, which can in turn be bounded by $\left(\Sigma_{K} \eta_{K}^{2}\right)^{1 / 2}$. The final result is that an adequate indicator for $\left|\dot{u}-\dot{u}_{h}\right|_{1, \Omega}$ is $\left(\eta_{K}^{2}+\xi_{K}^{2}\right)^{1 / 2}$. In other words, for the refinement procedure one must use not only $\xi_{K}$, related to $\dot{u}(p)$, but also the error indicator for the solution $u(p)$ itself, $\eta_{K}$.

\section{Sensitivity of cost functions or constraints}

In most cases, it is not the derivative $\dot{u}$ what matters but instead the sensitivity of some real objective or cost function $\Psi$. Also, in problems of optimal design, the derivatives of the so-called inequality constraints 
are needed during the optimization process. We are thus faced with a real function $\Psi(p, v)$, defined over $\mathbf{R} \times H_{0}^{1}(\Omega)$ (or some suitable subset), and we must evaluate its derivative with respect to $p$ with $v$ subject to be the solution, $u(p)$ of the Poisson problem (1) or (2). We will denote by $\psi$ the composed function

$$
\psi(p)=\Psi(p, u(p))
$$

In this paper we are dealing with the so-called direct method for evaluating $\dot{\psi}$. Its derivation is straightforward: Differentiating (12) we obtain

$$
\dot{\psi}(p)=D_{1} \Psi(p, u(p))+\left[D_{2} \Psi(p, u(p))\right] \cdot \dot{u}(p)
$$

where $D_{i}$ denotes the partial derivative with respect to the $i$-th variable, and

$$
\left[D_{2} \Psi(p, u(p))\right] \cdot \dot{u}(p) \stackrel{d e f}{=} \lim _{\alpha \rightarrow 0} \frac{1}{\alpha}[\Psi(p, u(p)+\alpha \dot{u}(p))-\Psi(p, u(p))]
$$

It should be clear that $D_{2} \Psi$ is a linear function from $H_{0}^{1}(\Omega)$ into R. From (13) and (9) or (3) the desired sensitivity $\dot{\psi}$ is obtained. In the numerical realization, $\dot{\psi}_{h}$ is computed using (13), with $u(p)$ and $\dot{u}(p)$ replaced by their approximations $u_{h}(p)$ and $\dot{u}_{h}(p)$.

\section{A posteriori error estimation for sensitivity of cost functions}

We now look for a suitable error indicator for $\dot{\psi}$, so that we can adapt the mesh and reduce the error $\left|\dot{\psi}-\dot{\psi}_{h}\right|$. Much of the work has already been done, we first use (13) to write

$$
\dot{\psi}(p)-\dot{\psi}_{h}(p)=D_{1} \Psi(p, u(p))-D_{1} \Psi\left(p, u_{h}(p)\right)+D_{2} \Psi(p, u(p)) \cdot \dot{u}(p)-D_{2} \Psi\left(p, u_{h}(p)\right) \cdot \dot{u}_{h}(p)
$$

The function $\Psi$ is now assumed to be twice continuously differentiable, its second derivative with respect to the $i$-th and $j$-th variables will be denoted by $D_{i j}^{2} \Psi$. Performing a Taylor series expansion we get

$$
\begin{gathered}
\dot{\psi}(p)-\dot{\psi}_{h}(p)=D_{12}^{2} \Psi\left(p, u_{h}(p)\right) \cdot\left(u(p)-u_{h}(p)\right)+D_{22}^{2} \Psi\left(p, u_{h}(p)\right):\left(\dot{u}_{h}(p), u(p)-u_{h}(p)\right)+ \\
+D_{2} \Psi\left(p, u_{h}(p)\right) \cdot\left(\dot{u}(p)-\dot{u}_{h}(p)\right)+\text { h.o.t. }
\end{gathered}
$$

where h.o.t. stands for terms which are of higher order in $u-u_{h}$ and $\dot{u}-\dot{u}_{h}$. Also, we have used the notation $D_{22}^{2} \Psi:(v, w)$ to denote the application of the bilinear form $D_{22}^{2} \Psi$ on the two elements $v$ and $w$ of $H_{0}^{1}(\Omega)$. It is now clear from the previous equation that, as the derivatives of $\Psi$ are bounded, if we reduce the error in $u$ and $\dot{u}$ we will automatically reduce the error in $\dot{\psi}$. So, in general, taking into account the actual form of the cost function $\Psi$ does not modify our indicator $\left(\eta_{K}^{2}+\xi_{K}^{2}\right)^{1 / 2}$.

If the error indicator $\left(\eta_{K}^{2}+\xi_{K}^{2}\right)^{1 / 2}$ is used, and thus a sequence of meshes is constructed with increasing accuracy for $u_{h}$ and $\dot{u}_{h}$, no problem should appear with the accuracy of $\dot{\psi}_{h}$. In fact, as proved by M.Masmoudi using an argument quite similar to the Aubin-Nitsche trick (see [7]), in many cases $\left|\dot{\psi}-\dot{\psi}_{h}\right|$ converges to zero not with $O\left(h^{k}\right)$ but with $O\left(h^{2 k}\right)$, where $k$ is the degree of the finite element space.

Remark: If the cost function $\Psi$ can be written in integral form, i.e.

$$
\Psi(u)=\int_{\Omega} g(u, \nabla u)
$$

where we have assumed for simplicity that there is no explicit dependence on the parameter, then the leading terms in the Taylor expansion of $\dot{\psi}-\dot{\psi}_{h}$ are

$$
\begin{aligned}
& \dot{\psi}-\dot{\psi}_{h} \simeq \int_{\Omega}\left[D_{1} g\left(\dot{u}-\dot{u}_{h}\right)+D_{2} g \cdot \nabla\left(\dot{u}-\dot{u}_{h}\right)+D_{11}^{2} g u_{h}\left(u-u_{h}\right)+\right. \\
& \left.+D_{22}^{2} g:\left(\nabla u_{h}, \nabla\left(u-u_{h}\right)\right)+D_{12}^{2} g \cdot\left(u_{h} \nabla\left(u-u_{h}\right)+\left(u-u_{h}\right) \nabla u_{h}\right)\right]
\end{aligned}
$$

As a consequence, if in some region of the domain one of the derivatives $D_{1} g, D_{2} g, D_{11}^{2} g, D_{22}^{2} g$ or $D_{12}^{2} g$ has a sharp peak, then the elements in this region should be refined to keep $\left|i^{1}-\psi_{h}\right|$ under control. As 
an informal example, if $g(u, \nabla u)=u^{m}$, with $m$ a positive integer, then $D_{1} g=m u^{m-1}, D_{2} g=0, D_{11}^{2} g=$ $m(m-1) u^{m-2}, D_{22}^{2} g=0$ and $D_{12}^{2} g=0$. Thus, if $m \gg 2$ it could be necessary to refine the mesh in regions where $|u|$ (in practice $\left|u_{h}\right|$ ) is large.

\section{EXTENSION TO LINEAR ELASTICITY}

The extension of the results of the previous section to linear problems in elasticity is straightforward. In this case, we must deal with the following differential problem: Find $u \in\left[H_{0}^{1}(\Omega)\right]^{n}$ such that

$$
\begin{gathered}
R(u(p))=\operatorname{div} \sigma(u(p))+f(p)=0 \\
\sigma(u)=\lambda(p)(\operatorname{div} u(p)) 1+2 \mu(p) \epsilon(u(p))
\end{gathered}
$$

holds in the weak sense, where $\left[H_{0}^{1}(\Omega)\right]^{n}$ is the space of square-integrable vector fields with square-integrable derivatives and zero trace on the boundary, $\lambda(p)$ and $\mu(p)$ the Lamé coefficients which could possibly have spatial variations and depend on the parameter $p, 1$ the identity tensor, $\epsilon(u)$ the strain (the symmetric part of the gradient of $u$ ), and $n$ the number of space dimensions.

The corresponding variational formulation is

$$
\int_{\Omega} \sigma(u(p)): \epsilon(v)=\int_{\Omega} f(p) \cdot v \quad \forall v \in\left[H_{0}^{1}(\Omega)\right]^{n}
$$

and the approximate solution $u_{h}(p)$ is obtained restricting this variational problem to a finite-dimensional subspace $V_{h}$ of $\left[H_{0}^{1}(\Omega)\right]^{n}$.

We need also to construct a differential equation for the derivative $\dot{u}(p)$, namely (ommiting the argument $p$ for brevity),

$$
Q(\dot{u})=\operatorname{div}(\lambda \operatorname{div}(\dot{u}) 1+2 \mu \epsilon(\dot{u}))+\left(\frac{d \lambda}{d p} \operatorname{div}(u) 1+2 \frac{d \mu}{d p} \epsilon(u)\right)+\frac{d f}{d p}=0
$$

where it is assumed that $u$ has been previously obtained from (15) and the only unknown is $\dot{u}$. Turning to the variational form of (17), it reads

$$
\int_{\Omega} \sigma(\dot{u}): \epsilon(v)=\int_{\Omega}\left[\frac{d f}{d p} \cdot v-\Sigma(u): \epsilon(v)\right] \quad \forall v \in\left[H_{0}^{1}(\Omega)\right]^{n}
$$

where we have introduced the operator

$$
\Sigma(u) \stackrel{\text { def }}{=} \frac{d \lambda}{d p} \operatorname{div}(u) 1+2 \frac{d \mu}{d p} \epsilon(u)
$$

The discrete problem to be solved in order to find the approximation $\dot{u}_{h}$ to $\dot{u}$ is the restriction of (18) to $V_{h}$ with $u$ in the right-hand side replaced by $u_{h}$. Higher order derivatives and its approximations are constructed in the same way.

The a posteriori error indicator for $u$, again of the Babuška-Miller type, is

$$
\eta_{K}^{2}=|K| \int_{K}\left|R\left(u_{h}\right)\right|^{2}+\sum_{l \in \partial K}|\eta| \int_{l}\left|\left[\sigma\left(u_{h}\right)^{+}-\sigma\left(u_{h}\right)^{-}\right] \mathbf{n}\right|^{2}
$$

and the error indicator we propose for $\dot{u}$ is again $\left(\eta_{K}^{2}+\xi_{K}^{2}\right)^{1 / 2}$ with

$$
\xi_{K}^{2}=|K| \int_{K}\left|Q\left(\dot{u}_{h}\right)\right|^{2}+\sum_{l \in \partial K}|l| \int_{l}\left|\left[\left(\sigma\left(\dot{u}_{h}\right)+\Sigma\left(u_{h}\right)\right)^{+}-\left(\sigma\left(\dot{u}_{h}\right)+\Sigma\left(u_{h}\right)\right)^{-}\right] \mathbf{n}\right|^{2}
$$

In Appendix B we include a mathematical result that supports the use of these indicators for error estimation. The proof is completely analogous to that of Appendix A for Poisson-type problems. 
Once the error indicator has been constructed, previous considerations of concerning the evaluation of derivatives of cost functions or constraints remain valid.

\section{THE POTENTIAL ENERGY AS COST FUNCTION}

One frequent design criterion is to minimize the total potential energy $\Pi(u)$, which for Poisson-type problems is given by

$$
\mathrm{\Pi}(u)=E(u)-T(u)=\frac{1}{2} \int_{\Omega} k|\nabla u|^{2}-\int_{\Omega} f u
$$

where $E(u)$ is the "internal energy" and $T(u)$ the "work of external actions". Let us show how the error in this cost function and in its sensitivity can be estimated in terms of the indicators $\eta_{K}$ and $\xi_{K}$ previously introduced.

Let $e=u-u_{h}, \dot{e}=\dot{u}-\dot{u}_{h}$. We already know that

$$
\begin{gathered}
|e|_{1, \Omega}^{2} \leq C \sum_{K \in \mathcal{T}_{h}} \eta_{K}^{2} \\
|\dot{e}|_{1, \Omega}^{2} \leq C \sum_{K \in \mathcal{T}_{h}}\left(\eta_{K}^{2}+\xi_{K}^{2}\right)
\end{gathered}
$$

Now notice that

$$
\begin{gathered}
E(u)-E\left(u_{h}\right)=\frac{1}{2} \int_{\Omega} k\left(|\nabla u|^{2}-\left|\nabla u_{h}\right|^{2}\right)=\frac{1}{2} \int_{\Omega} k\left(\nabla u-\nabla u_{h}\right)\left(\nabla u+\nabla u_{h}\right)= \\
=E\left(u-u_{h}\right)+\int_{\Omega} k \nabla e \nabla u_{h}=E\left(u-u_{h}\right)
\end{gathered}
$$

The last equality follows from the orthogonality of the error. We have then

$$
E(u)-E\left(u_{h}\right)=\frac{1}{2} \int_{\Omega} k|\nabla e|^{2} \leq C|e|_{1, \Omega}^{2} \leq \tilde{C} \sum_{K \in T_{h}} \eta_{K}^{2}
$$

Similarly, for $T$ we have

$$
T(u)-T\left(u_{h}\right)=\int_{\Omega} f e=\int_{\Omega} k \nabla u \nabla e=\int_{\Omega} k|\nabla e|^{2} \leq C|e|_{1, \Omega}^{2} \leq \dot{C} \sum_{K \in T_{\Lambda}} \eta_{K}^{2}
$$

and thus

$$
\left|\Pi(u)-\Pi\left(u_{h}\right)\right| \leq C \sum_{K \in T_{h}} \eta_{K}^{2}
$$

Consider now the sensitivity of the potential energy. For İ we get

$$
\begin{gathered}
\left|\dot{E}(u)-\dot{E}\left(u_{h}\right)\right|=\left.\left|\frac{1}{2} \int_{\Omega} \frac{d k}{d p}\right| \nabla e\right|^{2}+\int_{\Omega} k \nabla e \nabla \dot{e} \mid \leq C\left(|e|_{1, \Omega}^{2}+2|e|_{1, \Omega}|\dot{e}|_{1, \Omega}\right) \leq \\
\leq C\left(|e|_{1, \Omega}^{2}+\frac{1}{\alpha}|e|_{1, \Omega}^{2}+\alpha|\dot{e}|_{1, \Omega}^{2}\right) \leq \dot{C} \sum_{K \in T_{h}}\left[\left(1+\alpha+\frac{1}{\alpha}\right) \eta_{K}^{2}+\alpha \xi_{K}^{2}\right] \\
\left|\dot{T}(u)-\dot{T}\left(u_{h}\right)\right|=\left|\int_{\Omega}\left(f \dot{e}+\frac{d f}{d p} e\right)\right|=\left|\int_{\Omega}\left(k \nabla u \nabla \dot{e}+k \nabla \dot{u} \nabla e+\frac{d k}{d p} \nabla u \nabla e\right)\right|= \\
=\left|\int_{\Omega}\left(k \nabla e \nabla \dot{e}+k \nabla \dot{e} \nabla e+\frac{d k}{d p} \nabla e \nabla e\right)\right| \leq C \sum_{K \in T_{h}}\left[\left(1+\alpha+\frac{1}{\alpha}\right) \eta_{K}^{2}+\alpha \xi_{K}^{2}\right]
\end{gathered}
$$

with $\alpha$ any positive number. In the derivation, we have used the property

$$
\int_{\Omega}\left(k \nabla \dot{e} \nabla v+\frac{d k}{d p} \nabla e \nabla v\right)=0 \quad \forall v \in V_{h}
$$


We have thus proved that

$$
\left|\dot{\Pi}-\dot{I}_{h}\right| \leq C \sum_{K \in T_{h}}\left[\left(1+\alpha+\frac{1}{\alpha}\right) \eta_{K}^{2}+\alpha \xi_{K}^{2}\right]
$$

Notice that this results predicts that the order of convergence of $\dot{\Pi}_{h}$ to $\dot{\Pi}$ doubles that of $\dot{u}_{h}$ to $\dot{u}$, in accordance with the a priori estimates of [7]. It does not seem possible to avoid the calculation of $\xi_{\kappa}$ (and thus of $\dot{u}_{h}$ ) without stronger regularity assumptions on $u$. This is quite surprising since, as is well-known, the evaluation of $\dot{\Pi}$ does not require the computation of $\dot{u}$.

Similar results can be found for linear elasticity.

\section{SHAPE SENSITIVITY}

We will show now how our error estimation procedure is to be used in shape optimization problems. Consider a "velocity" field $V$ defined on $\Omega$, such that the perturbations in shape to be considered are obtained moving $x \in \Omega$ to $x+p V(x)$. The deformed domains will be labeled $\Omega+p V$. We first define the family $U_{p}$ of solutions of the Poisson problem

$$
\begin{aligned}
-\nabla \cdot\left(\kappa \nabla U_{p}\right)=q & \text { in } \quad \Omega+p V \\
U_{p}=0 & \text { on } \partial(\Omega+p V)
\end{aligned}
$$

and now introduce $u_{p}$ as the translation of $U_{p}$ to $\Omega$ by

$$
u_{p}(x)=U_{p}(x+p V(x))
$$

It is well-known that $u_{p}$ satisfies the variational equation

$$
\int_{\Omega} \kappa\left(\mathbf{G}_{p}^{-1} \nabla u_{p}\right) \cdot\left(\mathrm{G}_{p}^{-1} \nabla w\right) \operatorname{det}\left(\mathbf{G}_{p}\right)=\int_{\Omega} q w \operatorname{det}\left(\mathbf{G}_{p}\right) \quad \forall w \in H_{0}^{1}(\Omega)
$$

where $G_{p}$ is the tensor

$$
\left(\mathrm{G}_{p}\right)_{i j}=\delta_{i j}+p \frac{\partial V_{j}}{\partial x_{i}}
$$

In shape optimization problems we need to evaluate $d u_{p} / d p(p=0)=\dot{u}$. But, from (27), we can immediately use the results of the second section, with $k(p)$ (now a tensor) and $f(p)$ given by

$$
\begin{aligned}
& k(p)=\operatorname{det}\left(\mathrm{G}_{p}\right) \kappa \mathrm{G}_{p}^{-T} \mathrm{G}_{p}^{-1} \\
& f(p)=\operatorname{det}\left(\mathrm{G}_{p}\right) q
\end{aligned}
$$

$d k / d p$ and $d f / d p$ are obtained by direct differentiation of these expressions. The error estimator of the second section can thus be applied without change, and the theoretical results in Appendix A remain valid. An a posteriori error estimator for shape sensitivity in elasticity can also be found analogously.

\section{FURTHER REMARKS AND CONCLUSIONS}

We have presented a posteriori error indicators for diffusion and elasticity problems, which can be used to refine the mesh in finite-element sensitivity analyses. To our knowledge, this is the first paper presenting a systematic approach to improve the accuracy in this kind of problems. Our methodology applies also to any linear problem for which a residual-based error indicator exists. The main steps to build the extra-term $\xi_{K}$ to be added to the original error indicator $\eta_{K}$ are:

1. Write down the differential equation for $\dot{u}$. 
2. Replace, in the expression of the error indicator $\eta_{K}$, the residual and jump terms so that the resulting $\xi_{K}$ is consistent with the differential equation for $\dot{u}$.

In nonlinear problems, as the differential equation for $\dot{u}$ does not in general contain the same differential operator as that for $u$, a suitable error indicator (instead of $\eta_{K}$ ) must be used in Step 2. Also, the algorithm can be easily extended to handle more general boundary conditions that those analysed in this paper.

From the theory, it is clear that the relative weights of $\eta_{K}$ and $\xi_{K}$ cannot be predicted. In fact, $\left(\eta_{K}^{2}+C \xi_{K}^{2}\right)^{1 / 2}$, with $C$ arbitrary, would yield the same theoretical bound. We thus propose, as a practical algorithm, to refine in those regions of the mesh where either $\eta_{K}$ or $\xi_{K}$ are large (with respect to their mean values). The remark in 2.4 should also be kept in mind.

We would like to finish this paper explaining why we have not included any numerical result. As our exposition was kept at a general level, numerical applications will be reported in future papers. The point is that it is quite trivial to show that the error indicator $\eta_{K}$ is insufficient to conveniently adapt the mesh in sensitivity analyses: Just consider the case $f(p)=p f_{0}, k(p)=k_{0}$ for the Poisson problem, with $f_{0}$ a given function in $L^{2}(\Omega)$ and $k_{0}$ a real number, and try to evaluate $\dot{u}(0)$. It is clear that $u(0)=u_{h}(0)=0$ and thus $\eta_{K}=0$ for every element $K$. Also, $\dot{u}(0)$ is in this case the solution of

$$
-k_{0} \Delta \dot{u}(0)=f_{0}
$$

which is not in general an element of $V_{h}$ and thus $\dot{u}_{h}(0)$, obtained from (5) will contain an error that is not seen by $\eta_{K}$. In fact, $\eta_{K}$ would say that any mesh is perfect!

\section{Appendix A}

In this appendix the rigorous proof of the equivalence between the true error and our error estimator is sketched, considering Poisson-type problems. It is as usual assumed that the triangulations of $\Omega$ are always regular (see [4]).

Theorem A1 (Error estimation for $u_{h}$ ): With the definitions and assumptions of the second section, there exist two constants $C_{1}$ and $C_{2}$ depending only on the minimum angle of $\tau_{h}$ and on the bounds of $k(p)=k(p, x)$ such that,

$$
C_{1}\left(\sum_{T \in T_{h}} \eta_{K}^{2}\right)^{\frac{1}{2}} \leq\left|u-u_{h}\right|_{1, \Omega} \leq C_{2}\left(\sum_{T \in T_{h}} \eta_{K}^{2}\right)^{\frac{1}{2}}
$$

Proof: The proof will not be given here because it is essentially identical to that in [9] ( see also [1]). 0 Lemma A1: For $v \in H_{0}^{1}$ and $v_{h} \in V_{h}$ we have the following error equation,

$$
\begin{aligned}
& \int_{\Omega} k \nabla\left(\dot{u}-\dot{u}_{h}\right) \nabla v+\int_{\Omega} \dot{k} \nabla\left(u-u_{h}\right) \nabla v= \\
& \sum_{T \in T_{\mathrm{h}}} \int_{T} Q\left(v-v_{h}\right)+\frac{1}{2} \sum_{\ell \in \partial T} \mid \| \int_{l} \tilde{J}_{\ell}\left(v-v_{h}\right)
\end{aligned}
$$

where $\tilde{J}_{l}=\left.\left[\left(k \nabla \dot{u}_{h}+(d k / d p) \nabla u_{h}\right)^{+}-\left(k \nabla \dot{u}_{h}+(d k / d p) \nabla u_{h}\right)^{-}\right] \cdot \mathbf{n}\right|_{\ell}$

Proof : Using the orthogonality of $u-u_{h}$ to $V_{h}$ and integrating by parts in each element we have

$$
\begin{aligned}
& \int_{\Omega} k \nabla\left(u-u_{h}\right) \nabla v+\int_{\Omega} k \nabla\left(u-u_{h}\right) \nabla\left(v-v_{h}\right)= \\
& \sum_{T \in \tau_{h}} \int_{T} R\left(v-v_{h}\right)+\frac{1}{2} \sum_{\ell \in \partial T}|l| \int_{l}\left[\left(k \nabla u_{h}\right)^{+}-\left(k \nabla u_{h}\right)^{-}\right] \cdot \mathbf{n}\left(v-v_{h}\right)
\end{aligned}
$$

and the lemma follows differentiating with respect to $p .0$

Theorem A2 (Error estimation for $\dot{u}_{h}$ ): With the definitions and assumptions of the second section, there exist two constants $C_{1}$ and $C_{2}$, depending only on the minimum angle of $\tau_{h}$ and on the bounds of $k(p)=k(p, x)$ such that,

$$
C_{1}\left\{\sum_{T \in T_{h}}\left(\eta_{K}^{2}+\xi_{K}^{2}\right)\right\}^{\frac{1}{2}} \leq\left|\dot{u}-\dot{u}_{h}\right|_{1, \Omega} \leq C_{2}\left\{\sum_{T \in T_{h}}\left(\eta_{K}^{2}+\xi_{K}^{2}\right)\right\}^{\frac{1}{2}}
$$

Proof : Let $w \in H_{0}^{1}$ be such that

$$
\int_{\Omega} k \nabla w \nabla v=\int_{\Omega} \dot{f} v-\int_{\Omega} \dot{k} \nabla u_{h} \nabla v \quad \forall v \in H_{0}^{1}
$$


We proceed to estimate $|\dot{u}-w|_{1, \Omega}$

$$
\alpha|\dot{u}-w|_{i, \Omega}^{2} \leq \int_{\Omega} k \nabla(\dot{u}-w) \nabla(\dot{u}-w)=-\int_{\Omega} \dot{k} \nabla\left(u-u_{h}\right) \nabla(\dot{u}-w) \leq C\left|u-u_{h}\right|_{1, \Omega}|\dot{u}-w|_{1, \Omega}
$$

where $\alpha=\inf _{\Omega} k$. Using Theorem Al we have

$$
|\dot{u}-w|_{1, \Omega} \leq C\left|u-u_{h}\right|_{1, \Omega} \leq \bar{C}\left(\sum_{T \in T_{A}} \eta_{T}^{2}\right)^{\frac{1}{3}}
$$

On the other hand, in view of the relation

$$
\int k \nabla\left(w-\dot{u}_{h}\right) \nabla v_{h}=0 \quad \forall v_{h} \in V_{h}
$$

we have that

$$
\alpha\left|w-u_{h}\right|_{1, \Omega}^{2} \leq \int_{\Omega} \nabla\left(w-\dot{u}_{h}\right)+\nabla\left[\left(w-\dot{u}_{h}\right)-\left(w-\dot{u}_{h}\right)^{l}\right]
$$

where $v^{I}$ is the Clement interpolated of $v$ [5]. Now, integrating by parts, we have that

$$
\alpha\left|w-\dot{u}_{h}\right|_{1, \Omega}^{2} \leq \sum_{T \in T_{h}} \int_{T} Q\left[\left(w-\dot{u}_{h}\right)-\left(w-\dot{u}_{h}\right)^{I}\right]-\sum_{\ell \in \partial T} \int_{l}\left(k \nabla u_{h}+k \nabla \dot{u}_{h}\right) \cdot \mathbf{n}\left[\left(w-\dot{u}_{h}\right)-\left(w-\dot{u}_{h}\right)^{I}\right]
$$

and using the usual properties of the Clement interpolation [5]

we obtain the right inequality of the theorem.

$$
\begin{gathered}
\left\|u-u^{I}\right\|_{L^{2}(T)} \leq C|T|^{\frac{1}{2}}\|\nabla u\|_{L^{2}(\bar{T})} \\
\left\|u-u^{I}\right\|_{L^{2}(\ell)} \leq C|\ell|^{\frac{1}{5}}\|\nabla u\|_{L^{2}(\bar{T})}
\end{gathered}
$$

To prove the other inequality we follow the ideas developed by Verfürth [9] (see also [1]). We use the error equation of Lemma Al with a particular choice of $v \in H_{\Gamma_{1}}^{1}$ and $\varphi \in H_{\Gamma_{2}}^{1}$ satisfying,

and

$$
\begin{array}{ll}
\int_{T} Q v=|T|\|Q\|_{L^{2}(T)}^{2} & , \forall T \in T_{h} \\
\int_{\ell} \tilde{J}_{\ell v}=|\ell|\left\|\tilde{J}_{\ell, n}\right\|_{L^{2}(\ell)}^{2} & , \forall \ell
\end{array}
$$

$$
|v|_{1, T} \leq C \eta_{T}
$$

It is not difficult to see that such $v$ and $\varphi$ exist. For example, if linear elements are used, $\varphi$ can be taken as a continuous piecewise quadratic polynomial vanishing at every vertex of the triangulation and $v$ as a continuous piecewise polynomial of degree three (in fact, quadratic augmented with local bubbles). We refer to [9] for the details. Now, Lemma A1 together with (A.1), (A.2) and (A.3) yields,

$$
\sum_{T \in T_{\AA}} \xi_{T}^{2}=\sum_{T \in T_{\AA}} \int_{T}(k \nabla \dot{e}+\dot{k} \nabla e) \cdot \nabla v \leq C\left(|e|_{1, \Omega}+|\dot{e}|_{1, \Omega}\right)\left\{\sum_{T \in T_{h}} \xi_{T}^{2}\right\}^{\frac{2}{2}}
$$

and using the theorem Al we have,

$$
\sum_{T \in T_{\Lambda}}\left(\eta_{T}^{2}+\xi_{T}^{2}\right) \leq C\left(|e|_{1, \Omega}+|\dot{e}|_{1, \Omega}\right)\left\{\sum_{T \in T_{\Lambda}}\left(\eta_{T}^{2}+\xi_{T}^{2}\right)\right\}^{\frac{3}{3}}
$$

which concludes the proof of the theorem. $\square$

\section{Appendix B}

We now state without proof (as it is analogous to that of Appendix A) the equivalence of the true error and the error estimator for elasticity problems. 
Theorem B1 : With the definitions and assumptions of the third section, there exist two constants $C_{1}$ and $C_{2}$, depending only on the minimum angle of $\tau_{h}$ and on the bounds of $\lambda$ and $\mu$ such that,

$$
C_{1}\left\{\sum_{T \in T_{h}}\left(\eta_{K}^{2}+\xi_{K}^{2}\right)\right\}^{\frac{1}{2}} \leq\left|u-u_{h}\right|_{1, \Omega}+\left|\dot{u}-\dot{u}_{h}\right|_{1, \Omega} \leq C_{2}\left\{\sum_{T \in T_{h}}\left(\eta_{K}^{2}+\xi_{K}^{2}\right)\right\}^{\frac{1}{2}}
$$

ACKNOWLEDGEMENTS: G.C.B. and C.P. also belong to Instituto Balseiro, Universidad Nacional de Cuyo and C.N.E.A., Argentina. Helpful discussions with Profs. J. S. Arora, E.Fancello and E. Taroco are gratefully acknowledged. This work was partially supported by Consejo Nacional de Investigaciones Científicas y Técnicas (CONICET), Argentina, and by Conselho Nacional de Desenvolvimento Científico e Tecnológico (CNPq), Brasil.

\section{References}

[1] I. Babus̄ka, R. Durán, R. Rodriguez, "Analysis of the efficiency of an a posteriori error estimator for linear triangular finite elements", SIAMJ. Numer. Anal. 29, 1992, pp 947-964.

[2] I.Babuška and A.Miller, "A posteriori error estimates and adaptive techniques for the finite element method" Technical Note BN-968, Inst. for Phys. Sci. and Technol., Univ. of Maryland, 1981.

[3] I.Babuška and D.Yu, "Asymptotically exact a posteriori error estimator for biquadratic elements", Technical Note BN-1050, Inst. for Phys. Sci. and Technol., Univ. of Maryland, 1986.

[4] P.G.Ciarlet, The Finite Element Method for Elliptic Problems, North Holland, 1978.

[5] P. Clément, "Approximation by finite elements function using local regularization", R.A.I.R.O., Anal. Numer. 7 R-2, 1975, pp. 77-84.

[6] H.R.E.Hornlein and K.Schittkowski (Eds.), Software Systems for Structural Optimization, Intl. Series of Numer. Math., Vol. 110, Birkhauser Verlag, Basel, 1993.

[7] P.Guillaume and M.Masmoudi, "Còmputation of higher order derivatives in optimal shape design", Nemerische Mathematik, to appear.

[8] C.Padra and M.J.Vénere, "On adaptivity for diffusion problems using triangular elements", Eng. Comp., to appear.

[9] R. Verfürth, "A posteriori error estimators for the Stokes equations", Numer. Math. 55, 1989, pp. 309-325. 